\title{
IMPORTANT INFECTIOUS DISEASES IN THE LAST DECADE
}

\section{Temenuga Stoeva}

\author{
Department of Microbiology and Virology, Medical University of Varna
}

Despite the remarkable advances in medical research and treatment during the $20^{\text {th }}$ century, infectious diseases remain among the leading causes of death worldwide. In a recent survey by the World Health Organization, three infectious diseases are ranked in the top ten causes of death globally - lower respiratory tract infections (3.1 million deaths; 5.5\%), HIV/AIDS (1.5 million deaths; $2.7 \%)$ and diarrheal diseases (1.5 million deaths; $2.7 \%)$. In low-income and lower-middle income countries, malaria and tuberculosis account for an additional two of the major ten causes of death and during the period 2014-2015 are responsible for 438000 and 1.5 million deaths, respectively (12). In addition to these, vaccine-preventable childhood diseases, meningitis, sexually transmitted infections (other than HIV), Hepatitis B and C, dengue and tropical disease also contribute significantly to the morbidity and mortality rates associated with infectious diseases (10).

During the last decade the European Center for Disease Prevention and Control (ECDC) monitors the epidemiology of more than 50 communicable diseases of public health importance in Europe and provides an annual picture and analysis of the state of these infections and threats detected in Europe. The major infectious diseases under surveillance are respiratory diseases, sexually transmitted infections (incl. HIV and blood born viruses), food and waterborne diseases, emerging and vectorborn, vaccine-preventable diseases, as well as antimicrobial resistance and healthcare-associated infections. The data from the last reports shows that respiratory tract infections, and particularly influenza, continue to cause a significant burden, especially to older individuals, very young children and subjects with chronic diseases. In the autumn of 2012 the first few cases in Europe of an emerging respiratory virus, that later became known as the Middle East respiratory syndrome coronavirus (MERS $\mathrm{CoV}$ ), were identified (3). Tuberculosis remains a common infection and an important public health problem in the EU, with more than 68000 cases reported annually. The highest rates are reported by Romania $(85.2$ per 100000 population), Lithuania (59.2), Latvia (48.6) and Bulgaria (31.1) (4). Regarding the STIs, infection with the bacterium Chlamydia trachomatis remains the most frequently reported sexually transmitted infection in Europe, where it is also the highest-ranking reportable disease. In 2012, 384105 cases of chlamydia were reported in $25 \mathrm{EU}$ Member States, an overall rate of 199 per 100000 population. Most chlamydial infections are asymptomatic and complications mostly affect women, including pelvic inflammatory disease, chronic pelvic pain and reduced fertility. In addition, Chlamydia trachomatis is a co-factor for HIV infection, increasing both susceptibility and infectiousness $(2,6)$. In this aspect, HIV infection remains a major public health concern in Europe. The overall reported incidence has stabilized at around 29000 cases annually, but reported rates continue to increase in many countries and vary widely between countries. The countries with the highest rates of HIV cases for 2014 are Estonia (23.6), Latvia (16.6), Belgium (11.1), Luxemburg (10.3) and the United Kingdom (10.1). The lowest rate is reported by Slovakia (0.9) (6). In the European context, the vector-borne diseases also pose a continuing and increasing public health risk. Locally acquired cases of diseases, previously considered to be only imported (malaria, dengue fever, chikungunya fever), continue to occur. The outbreak in Madeira in 2012 is the first major outbreak of dengue fever (with over 2000 cases) in the recent history, in an EU country, and illustrates the potential for local transmission where competent insect vectors are present (2). Antimicrobial resistance in association with hospital-acquired infections is another major public health problem in Europe. It is estimated that within the EU healthcare system, 4.2 million patients each year are

Scripta Scientifica Medica, vol. 48, No 1, 2016, pp. 7-8

Copyright (@) Medical University of Varna 
affected by healthcare-associated infections (HAIs). The main types of HAIs are respiratory tract infections (31.2\%), urinary tract infections (31.2\%) and skin infections (22.8\%) (5).

Among the major non-communicable diseases, cancer is a leading cause of morbidity and mortality worldwide, with approximately 14 million new cases and 8.2 million cancer-related deaths in $2012(9,11)$. It is expected that annual cancer cases will rise from 14 million in 2012 to 22 million within the next 2 decades (1). Specific infections are recognized as a major cause of cancer, with an estimated 2 million (16.1\%) of the 12.7 million new cases of cancer in 2008 attributable to infection (11). The most important infectious agents are Helicobacter pylori, Hepatitis B and C viruses and Human papillomaviruses, which together are responsible for 1.9 million cases of gastric, liver and cervix uteri cancers, respectively (1). In addition to these infectious agents, Opisthorchis viverrini, Clonorchis sinensis, Epstein-Barr virus, human T-cell lymphotropic virus type 1, human herpes virus type 8 (known as Kaposi's sarcoma herpes virus) and Schistosoma haematobium are classified as carcinogenic to humans.

As a reference to the significance of the infectious diseases nowadays, in this current issue of the journal, T. Kostadinova (7) and E. Kovachev (8) are focusing on two medically important microbial agents - Chlamydia trachomatis and Epstein-Barr virus. T. Kostadinova presents the current knowledge about the mechanisms of EBV pathogenesis in humans and its oncogenic potential. E. Kovachev reveals the relationship between Chlamydia trachomatis infection and pregnancy complications.

In conclusion, in the next decade the infectious diseases (incl. new and re-emergence of old infectious diseases) are expected to remain a serious challenge for detection and monitoring, prevention, control and adequate treatment.

\section{REFERENCES}

1. de Martel C, Ferlay J, Franceschi S, Vignat J, Bray F, Forman D, Plummer M. Global burden of cancers attributable to infections in 2008: a review and synthetic analysis. The Lancet Oncology 2012; 13: 607-615.

2. European Centre for Disease Prevention and Control. Annual Epidemiological Report 2013. Reporting on 2011 surveillance data and 2012 epidemic intelligence data. Stockholm: ECDC; 2013.

3. European Centre for Disease Prevention and Control. Annual epidemiological report 2014 - Respiratory tract infections. Stockholm: ECDC; 2014.

4. European Centre for Disease Prevention and Control. Annual epidemiological report 2014 - Respiratory tract infections - tuberculosis. Stockholm: ECDC; 2015.

5. European Centre for Disease Prevention and Control. Annual epidemiological report 2014 - Antimicrobial resistance and healthcare-associated infections. Stockholm: ECDC; 2015.

6. European Centre for Disease Prevention and Control. Annual epidemiological report 2014 - sexually transmitted infections, including HIV and blood-borne viruses. Stockholm: ECDC; 2015.

7. B. W. Stewart, C. P. Wild. World Cancer Report 2014.

8. WHO (2004) The world health report 2004 - changing history (http://www.who.int/whr/2004/en/)

9. http://www.iarc.fr

10. http://www.who.int/mediacentre/factsheets/fs310/en/). 